\title{
Soluble Ultra-Short Single-Walled Carbon
}

\section{Nanotubes-Supporting Information}

\author{
Zheyi Chen, Kazufumi Kobashi, Urs Rauwald, Richard Booker, Hua Fan, \\ Wen-Fang Hwang ${ }^{*}$ and James M. Tour ${ }^{*}$
}

Smalley Institute for Nanoscale Science and Technology, Carbon Nanotechnology Laboratory, Department of Chemistry, Rice University, 6100 Main Street, MS-100, Houston, Texas 77005, USA.

Email: whwang@rice.edu; tour@rice.edu

\section{References:}

21. Ericson, L. M.; Fan, H.; Peng, H.; Davis, V. A.; Zhou, W.; Sulpizio, J.; Wang, Y.; Booker, R.; Vavro, J.; Guthy, C.; Parra-Vasquez, A. N. G.; Kim, M. J.; Ramesh, S.; Saini, R. K.; Kittrell, C.; Lavin, G.; Schmidt, H.; Adams, W. W.; Billups, W. E.; Pasquali, M.; Hwang, W.-F.; Hauge, R. H.; Fischer, J. E.; Smalley, R. E. Science 2004, 305, 1447-1450. 